\title{
Fit für die digitale Disruption werden
}

\author{
Kurt Matzler · Franz Bailom
}

Online publiziert: 15. April 2019

(C) Der/die Autor(en) 2019

Zusammenfassung Die Autoren beschreiben, wie sich die digitale Transformation in Unternehmen auf Ebene der Produkte, der Prozesse und ganzer Geschäftsmodelle auswirkt, wie sich Wertschöpfungslogiken verändern und wie ein Mehrwert durch Digitalisierung geschaffen werden kann. Anhand von Beispielen aus unterschiedlichen Branchen entwickeln sie sieben Ratschläge, wie Unternehmen, sich auf die Veränderungen einstellen und ihr Geschäftsmodell erneuern können.

Schlüsselwörter Digitale Transformation · Internet der Dinge · Erfolgsfaktoren der Digitalisierung

\section{Getting ready for digital disruption}

\begin{abstract}
The authors describe how the digital transformation affects products, processes, and whole business models in organizations, how digitalization changes value creation mechanisms and how companies can add value for their customers. Using examples from different industries, they develop seven suggestions for adapting to the coming wave of digitalization and for the renewal of business models.
\end{abstract}

Keywords Digital transformation - Internet of things $\cdot$ Success factors of digitalization

Der vorliegende Beitrag basiert auf dem Buch: Matzler, K., Bailom, F., Friedrich von den Eichen, S., \& Anschober M. (2016). Digital Disruption. Wie Sie Ihr Unternehmen auf das digitale Zeitalter vorbereiten. München: Vahlen.

\footnotetext{
K. Matzler $(\bowtie)$

Universität Innsbruck, Universitätsstraße 15, 6020 Innsbruck, Österreich

E-Mail: Kurt.matzler@uibk.ac.at

Dr. F. Bailom

in-manas: intelligent management solutions $\mathrm{GmbH}$, Bienerstr. 4, 6020 Innsbruck, Österreich

E-Mail: f.bailom@in-manas.com
} 


\section{Digitale Transformation}

Disruption scheint wohl das Managementwort des Jahres zu sein (vgl. Meck und Weiguny 2015). Keine Vorstandssitzung ohne diesen Begriff. Start-ups werden im Silicon Valley gegründet - und nicht nur dort - mit dem Ziel, ganze Branchen zu zerstören. Der ehemalige CISCO-Chef prognostiziert, dass $40 \%$ der heutigen Unternehmen aufgrund der Digitalisierung in zehn Jahren verschwunden oder bedeutungslos sein werden - trotz aller Renovierungsbemühungen. Europa hinkt hinterher. Eine McKinsey-Studie behauptet, dass bislang nur 12\% des Digitalisierungspotenzials genutzt werden, in den USA sind es 18\% (Bughin et al. 2016)! Die erste Runde der digitalen Transformation ging an das Silicon Valley. Mit atemberaubender Geschwindigkeit verändern neue Technologien das gesamte Wirtschaftsgefüge, die Gesellschaft und die Art, wie wir leben, arbeiten und konsumieren.

Die digitale Transformation übertrifft alles Dagewesene an Entwicklungen hinsichtlich Schnelligkeit, Reichweite und systemischer Wirkung. Dazu eröffnet die Kombinatorik einzelner Technologien ungeahnte neue Möglichkeiten: Cloudtechnologien, künstliche Intelligenz, Rechnerleistung, Robotik, 3D-Druck, Sensorik, Big Data, Vernetzung usw. und deren Kombinationen führen in vielen Branchen zu vollkommen neuartigen Produkten, Dienstleistungen und Geschäftsmodellen. Die Herausforderungen sind gewaltig. Viele der digitalen Veränderungen sind disruptiv. Sie verändern Branchen grundlegend. Neue Geschäftsmodelle lösen alte $a b-$ in immer kürzeren Zeitabständen. Viele Unternehmen haben damit ihre Schwierigkeiten. Sie unterschätzen die Dynamik, reagieren zu langsam. Und sie halten an ihren bestehenden Geschäftsmodellen fest. In der Regel sind es Neueinsteiger und Startups, deren disruptive Geschäftsmodelle Branchen verändern oder gar überflüssig machen. Die digitale Transformation erfordert es, das gesamte Geschäftsmodell neu zu denken (Matzler et al. 2016).

Dabei spielt das Internet der Dinge eine entscheidende Rolle. Es bedeutet die Verbindung der physischen mit der digitalen Welt. Es verbindet physische Objekte, erlaubt Datengenerierung in Echtzeit, führt über Data Analytics zu Mustererkennung, erlaubt durch Anwendung von Algorithmen und maschinellem Lernen automatisierte Entscheidungen und Prozesse, Vorhersagen und Steuerung in Echtzeit. Eine McKinsey-Studie schätzt, dass die ökonomische Bedeutung des Internet der Dinge bis in das Jahr 2025 zwischen vier und elf Billionen Dollar liegen könnte - das wären etwa $11 \%$ der Weltwirtschaftsleistung (Manyika 2015). Das Internet der Dinge wird Treiber von neuen Geschäftsmodellen und Ökosystemen mit vier Merkmalen (vgl. Evans und Forth 2015):

- Internet der Dinge: Über 50 Mrd. mit dem Internet verbundene Dinge werden im Jahre 2020 existieren.

- Mobiles Internet: 6Mrd. Smartphones und 70\% der Weltbevölkerung werden bis 2020 einen mobilen Internetzugang haben (Schätzung von Ericsson 2015).

- Big Data: Die weltweit generierte Menge an verfügbaren Daten verdoppelt sich alle zwei Jahre, immer mehr Menschen liefern Daten und haben Zugang zu diesen Daten. 


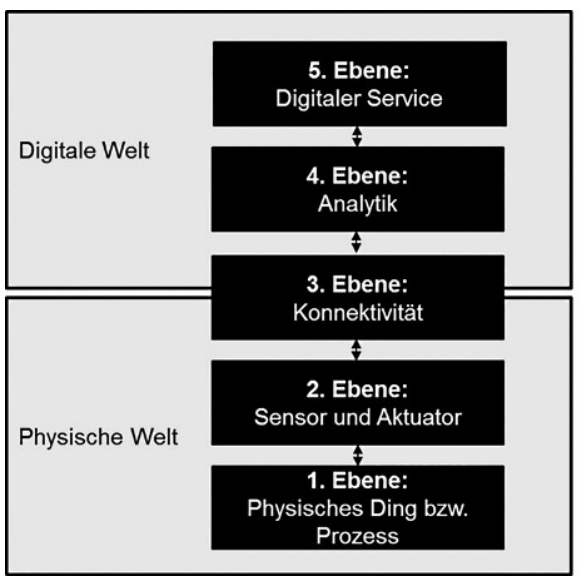

5. Welchen Mehrwert bzw. Kundennutzen

bieten diese Daten?

4. Welche Muster und Erkenntnisse lassen sich durch die Data Analytics finden?

3. Welchen Mehrwert liefert Konnektivität bzw. Daten in Echtzeit?

2. Welche sinnvollen Daten lassen sich durch Sensoren generieren? Welchen Nutzen können Aktuatoren liefern?

1. Welchen Mehrwert bzw. Kundennutzen liefert das digitalisierte Produkt oder der Prozess?

Abb. 1 Wertschöpfungslogiken im Internet der Dinge (in Anlehnung an Fleisch et al. 2014)

- Eine Revolution in Algorithmen, Maschinenlernen und statistischen Rückschlüsse wird diese Daten synthetisieren, zu neuen Erkenntnissen, verbesserten Dienstleistungen und neuen Geschäftsmodellen führen.

Die Kernfrage dabei lautet: Wie können physische Produkte oder Prozesse durch Digitalisierung Mehrwert schaffen oder Effizienz steigern? Abb. 1 stellt eine Wertschöpfungslogik der Digitalisierung dar. Es visualisiert, wie Digitalisierung, Konnektivität, Analytik und digitaler Service zu neuen Wertschöpfungs- und Ertragsquellen führen können. Sechs Fragen sind dabei entscheidend:

1. Welchen Mehrwert bzw. Kundennutzen liefert das Produkt oder der Prozess?

2. Welche sinnvollen Daten lassen sich durch Sensoren generieren?

3. Wie können diese Daten in Echtzeit gesammelt und mit anderen Daten verknüpft werden?

4. Welche Muster und Erkenntnisse lassen sich durch die Verknüpfung finden?

5. Welchen Mehrwert bzw. Kundennutzen bieten diese Daten?

6. Welche neuen Ertragsquellen lassen sich durch Digitalisierung erschließen?

\section{Mehrwert durch Digitalisierung}

Am Beispiel von Schindler, dem Schweizer Hersteller von Aufzügen, zeigen wir, wie durch Digitalisierung der Produkte und der Prozesse Mehrwert geschaffen und die Effizienz gesteigert werden können (vgl. Buvat und Subrahmanyam 2016).

1. Ebene: Das physische Ding bzw. der Prozess. Welchen Mehrwert bzw. Kundennutzen liefert das Produkt oder der Prozess? Die erste Ebene der Wertschöpfungslogik bildet das physische Ding bzw. der Prozess. Im Falle Schindler sind es Personenaufzüge. Ausgangspunkt für die Digitalisierung ist die Frage: „Wel- 
chen Mehrwert bzw. Kundennutzen liefert das Produkt bzw. der Prozess?“ und „Wie kann Digitalisierung diesen Mehrwert erhöhen?“"Im Fall von Aufzügen sind es Themen wie Komfort, Sicherheit, Effizienz, Energieverbrauch usw., die Differenzierungsmerkmale für einen Aufzughersteller darstellen. Schindlers Vision ist „Leadership through Customer Service“, mit technologischer Innovation als treibende Kraft. Dies Vision stellt die Richtung und die Leitplanken für das Digitalisierungsprojekt dar, ,Superior Customer Experience“ ist das Ziel.

2. Ebene: Sensor und Aktuator. Welche sinnvollen Daten lassen sich durch Sensoren generieren? Physische Objekte werden mit Sensoren und Aktuatoren ausgestattet. Sensoren liefern Daten in Echtzeit und erlauben sofortige Eingriffe zur Aufrechterhaltung des Produktionsflusses und Fehlerbehebung. Im Falle der Aufzüge von Schindler sind es Sensoren, die Parameter wie Vibration, Geschwindigkeit und Temperatur erfassen und in Echtzeit übermitteln.

3. Ebene: Konnektivität. Wie können diese Daten in Echtzeit gesammelt und mit anderen Daten verknüpft werden? Sind die physischen Objekte mit IP-fähigen Sensoren ausgestattet, können sie mit anderen Objekten kommunizieren bzw. Daten mitteilen. Mehr als 200 Mio. Datenpunkte werden bei Schindler in einem Control Center gesammelt. Daten alleine sind allerdings wertlos, wenn sie nicht entsprechend analysiert werden können. Daher kommen Algorithmen und Maschinenlernen zum Einsatz. Darin sieht Schindler heute einen entscheidenden Wettbewerbsvorteil.

4. Ebene: Analytik. Welche Muster und Erkenntnisse lassen sich durch die Verknüpfung finden? Über die Sammlung, Speicherung und Auswertung von Sensordaten lassen sich nun wertvolle Informationen gewinnen, die in Dienstleistungen mit Mehrwert transformiert werden können. Schindler nutzt Algorithmen und Maschinenlernen für vorausschauende Instandhaltung (predictive maintenance), automatische Generierung von Arbeitsaufträgen für Techniker, automatische Zuteilung von Liften usw. Dadurch entsteht Mehrwert für den Kunden und höhere Effizienz in den Prozessen.

5. Ebene: Digitale Dienstleistungen. Welchen Mehrwert bzw. Kundennutzen bieten diese Daten? Nun lassen sich auf Basis der Analytik digitale Dienstleistungen schnüren. Da Schindler Daten in Echtzeit hat, weiß der Aufzughersteller von Problemen mit den Aufzügen in der Regel, noch bevor es der Kunde weiß, der Service ist im Schnitt um 22\% schneller. Durch Predictive Maintenance lassen sich Stillstandszeiten weitgehend vermeiden. Und ein intelligentes Zuteilungssystem z. B. in großen Bürogebäuden, in denen sich Mitarbeiter mit Keycards identifizieren, sorgt dafür, dass Wartezeiten um $50 \%$ reduziert werden. Es entsteht als Mehrwert für den Kunden. Die so generierten Daten werden aber auch dazu verwendet, die Prozesse zu automatisieren bzw. zu beschleunigen. Schindlers Monteure und Servicetechniker (fast $60 \%$ der Gesamtbelegschaft) sind über die DIGITAL TOOL CASE digital vernetzt. Das System generiert automatisch Arbeitsaufträge in Echtzeit auf Basis der Liftdaten, es erstellt automatisch den Mitarbeitern Arbeitsanweisungen (Material, Werkzeug) und berechnet den schnellsten Weg zum Kunden. Techniker haben Zugang zu Multimediadokumenten mit Tipps und Richtlinien für die Reparatur, sie haben online Zugang zum Lager von 40.000 Teilen und Zugang zu allen Liftdaten in Echtzeit. 


\section{Erfolgsfaktoren für Digitalisierung}

Die Digitalisierung wirkt auf drei Ebenen: Auf die Produktebene, auf die Prozessebene und auf die Ebene des Geschäftsmodells. Vor allem die Verfügbarkeit von Daten und der daraus ableitbaren Dienstleistungen werden zu vielen neuen Geschäftsmodellen führen. Die Schwierigkeit in der digitalen Transformation liegt meist weniger in der Technologie. Natürlich müssen Technologien beherrscht und richtig eingesetzt werden. Natürlich geht es darum, die Dinge richtig zu tun - um mit Peter Drucker (1963) zu sprechen. Mindestens ebenso wichtig ist es allerdings auch, die richtigen Dinge zu tun. Hier sind aus unseren Erfahrungen sieben Punkte entscheidend:

1. Das Kundenproblem verstehen. Ein erfolgreiches Digitalisierungsprojekt beginnt beim Kunden. Was ist der „Job to be done“? Welches Problem des Kunden lösen wir, und wie können wir das digital noch besser machen? Wie können wir aus den „Painpoints“ des Kunden „Gainpoints“ für den Kunden machen? Eine sorgfältige Analyse der Customer Journey ist dabei hilfreich. Vielleicht haben Sie einen dieser altmodischen Hometrainer bei sich zu Hause stehen, auf dem Sie nur mit sehr viel Überwindung trainieren. Stellen Sie sich die Frage, was die „Painpoints“ des Kunden sind, dann finden Sie leicht Ansatzpunkte, wie Sie aus diesem langweiligen Produkt durch Digitalisierung ein spannendes machen können. Die „Painpoints“ sind: 1. Es ist langweilig, darauf zu trainieren, 2. Die Sitzposition ist unnatürlich, 3. Sie haben kaum Motivation, darauf lange zu trainieren. Taxc, ein Hersteller von Rollentrainern, hat dies erkannt. Der digitalisierte Rollentrainer kann an ein Fernsehgerät oder an ein Laptop angeschlossen werden. Sie können Videos z. B. von schönen Alpenpässen herunterladen und diese Strecken abfahren; wird es steil, steigt der Tretwiderstand; wenn jemand anderer die gleiche Strecke fährt, sehen Sie ihn und Sie können gegeneinander antreten; Sie können Ihre Leistungsdaten hochladen und online analysieren; Sie können sich Trainingspläne erstellen und Ihre Trainingsdaten auf Strava teilen und mit Ihren Freunden vergleichen. Aus einem langweiligen Produkt wird nicht nur ein spannendes, sondern der Anbieter hat durch ein Abomodell auch eine neue Ertragslogik gefunden.

2. Das richtige Bewusstsein entwickeln. Nach wie vor sind sich viele Unternehmen der disruptiven Gefahren gar nicht bewusst. „Wie würde Silicon Valley mein Geschäft zerstören, wen es das wollte?“, lautet die zentrale Frage. Viele Vorstandchefs reisen zurzeit ins Silicon Valley, um sich dieser Frage zu stellen. So auch Gisbert Rühl, CEO von Klöckner, Europas größtem Stahlhändler (vgl. Keese 2014). Nach einigen Firmenbesuchen und Gesprächen mit Internet-Gründern war die Antwort klar: Eine elektronische Plattform könnte den Todesstoß geben, ein Amazon der Stahlindustrie! Denken in Risiken kann zunächst sehr hilfreich sein. Es macht sensibel für die Gefahren, zeigt disruptive Bedrohungen auf und mobilisiert. Wer in Risiken denkt, muss aus der Komfortzone. Das größte Risiko, um mit Marc Zuckerberg zu sprechen, ist es, kein Risiko einzugehen: „Disrupt or be disrupted!" Um sich selbst zu erneuern - und zwar rechtzeitig -, müssen Unternehmen Bereitschaft zeigen, sich selbst zu zerstören, zumindest gedanklich, bevor es andere dann physisch tun. Das schließt die Bereitschaft mit ein, eigene Produkte, eigene Dienstleistungen oder gar das eigene Geschäftsmodell zu kanni- 
balisieren. Eine Reise ins Silicon Valley ist dabei gar nicht unbedingt nötig. Hier haben wir mit dem „Nightmare Competitor"-Ansatz gute Erfahrungen gemacht. Setzen Sie sich mit einem fiktiven Wettbewerber auseinander, der sich bestens mit der Zukunft arrangiert, alle Hebel der Digitalisierung zieht und das Geschäft nach ganz neuen Regeln betreibt und Ihnen so wirklich gefährlich werden kann. Diese Auseinandersetzung hilft, die Energie der kreativen Verzweiflung zu bündeln, zeigt Gefährdungspotenziale auf, hilft aber vor allem, rechtzeitig neue Geschäftslogiken zu finden. Ein Blick von außen nach innen, d.h. die Einbindung Externer, ist dabei nicht nur hilfreich, sondern dringend zu empfehlen.

3. In gesamten Geschäftsmodellen denken. Wer nicht in ganzen Geschäftsmodellen denkt, produziert Insellösungen. Es reicht nicht, nur einzelne Dimensionen zu verändern. Industrie 4.0 wird Unternehmen effizienter machen. Eine Studie der BCG prognostiziert, dass Produktionssysteme bis zu $30 \%$ schneller und $25 \%$ effizienter werden (BCG 2015). Das sind deutliche Kostenvorteile, und sie sind auch wichtig. Sie werden dazu beitragen, dass Unternehmen wettbewerbsfähig bleiben, und sie werden auch dazu beitragen, dass Teile der Produktion von Niedriglohnländern wieder zurück in Industrieländer verlagert werden - was bereits in vollem Gange ist. Diese Denkweise konzentriert sich zu stark auf Effizienzgewinne. Digitalisierung ist aber sehr viel mehr. Das Thema lediglich auf Effizienzsteigerung zu reduzieren, halten wir für einen Fehler. Die Effizienzgewinne nehmen uns in eine kreative Pflicht. Um das Beschäftigungsvakuum zu füllen, das durch Automatisierung, Robotik, künstliche Intelligenz usw. entstehen wird, müssen wir mehr über neue digitale Produkte und Dienstleistungen und neue Geschäftsmodelle nachdenken. Am Ende geht es um das (Er-)Finden neuer Wachstumsräume und das Kreieren digitaler Geschäftsmodelle. Nur so können wir Wachstum erzielen. Die Fragen, die sich dabei stellen, sind: Welche neuen Zielgruppen und welche neuen einzigartigen Nutzenversprechungen ermöglichen die digitalen Technologien und Entwicklungen? Welche neuen Produkte und Dienstleistungen ermöglichen die Digitalisierung? Wie verändern digitale Technologien meine Wertschöpfungslogiken und Prozesse? Welche neuen Vermarktungslogiken entstehen durch Digitalisierung? Wer aber bei der Digitalisierung noch ganz am Beginn steht, sollte seine Organisation nicht überfordern. Es gilt die 80/20-Regel: Identifizieren Sie jene Prozesse oder Bereiche, in denen Sie mit geringem Mitteleinsatz die größte Wirkung erzielen. Die Kundenschnittstelle bietet sich geradezu an. Beginnen Sie mit kleinen Projekten, die Ihnen „Quick Wins“ erlauben, verlieren Sie aber nicht das ganze Bild aus den Augen.

4. Den Strategieprozess öffnen. Niemand kann heute sagen, woher die nächste große Idee kommen wird. Daher müssen Unternehmen sich in ihren Strategieprozessen - bis zu einem gewissen Grad - öffnen. Die Schwarmintelligenz innerhalb und außerhalb des Unternehmens gilt es zu nutzen. Das Rad muss niemand neu erfinden. Schließlich sind $90 \%$ der Geschäftsmodellinnovationen nichts anderes als das Übertragen oder Rekombinieren von Mustern aus anderen Branchen (Gassmann et al. 2013). Große Ideen entstehen durch „Cross-Fertilization“, meistens dann, wenn man die Grenzen der eigenen Branche, der eigenen Expertise oder der eigenen Disziplin verlässt. Dabei ist es oft keineswegs nötig, die eigene Kernkom- 
petenz zu verlassen. Vielmehr geht es darum, diese mit anderen Technologien, anderem Wissen usw. zu verbinden.

5. Schnellboote entwickeln statt Tanker umbauen. Die meisten Unternehmen haben die größten Schwierigkeiten, wenn sie versuchen, neue digitale Geschäftsmodelle von innen heraus zu entwickeln - vor allem, wenn sie disruptiv sind. Sofort wird das Immunsystem des Unternehmens aktiviert. Die Angst vor der eigenen Kannibalisierung verhindert vieles. Die etablierten Strukturen und Prozesse lassen Flexibilität, Geschwindigkeit und Risiko nicht zu. Daher lohnt es sich oft, außerhalb der Unternehmensgrenzen zu beginnen. Der Stahlhändler Klöckner hat mit Klöckner.i ein eigenes Start-up in Berlin gegründet. Innerhalb der bestehenden Organisation räumt man dem Neuen kaum Umsetzungschancen ein. Dazu Gisbert Rühl (2016) in einem Handelsblatt-Interview: „Ich würde es immer wieder über eine separate Einheit machen. Mit Leuten von außen, die zunächst unabhängig arbeiten. In einem zweiten Schritt müssen Sie dafür sorgen, dass eine solche Einheit auf die übrige Organisation ausstrahlt und sich nicht als unabhängiger Satellit am Rande des Konzern-Orbits bewegt. Was wir versuchen, ist, die Start-up-Mentalität zu übertragen ...".

6. Mit Start-ups kooperieren. Das Engagement in Start-ups kann durchaus ein Weg sein, um in der Digitalisierung Fuß zu fassen. Start-ups funktionieren anders als etablierte Unternehmen. Sie folgen bedingungslos einer Idee, sind agil, zeigen Risikobereitschaft, bergen oftmals große Wachstumsaussichten - alles Eigenschaften, die etablierten Großunternehmen vielfach fehlen. Umgekehrt haben große Unternehmen Ressourcen, besondere Fähigkeiten, Stabilität, Macht, einen Markt und entsprechende Routinen, um Geschäftsmodelle effizient zu betreiben. Gelingt ein Zusammenwirken, können sich etablierte Unternehmen und Start-ups angesichts der komplementären Profile gut ergänzen. Mit Kooperationsprojekten erhoffen sich daher viele Unternehmen Zugang zu neuen Ideen und Zugang zu neuen Produkten, Einblick in die Szene rund um Gründer, Innovationen und Unternehmertum, von Start-ups und deren ,,way of business“ zu lernen und schließlich Investitionschancen. Mit Corporate Venturing, Corporate Accelerator-Programmen oder Corporate Incubation stellen sich vor allem große Unternehmen neu auf, um mehr Zugänge zur digitalen Szene zu bekommen.

7. Eine Fehlerkultur entwickeln. Schließlich brauchen wir eine neue Kultur, um die Innovationsfähigkeit zu entfesseln. Wir brauchen große Anreize für erfolgreiche Innovationen und keine Strafen für Fehler. In den meisten Unternehmen finden wir genau das Gegenteil vor. Im Silicon Valley herrscht der Grundsatz: schneller Fehler zu machen, um schneller zu lernen!

Digitalisierung ist - wie erwähnt - weniger eine Frage der Beherrschung einzelner Technologien. Zahlreiche Technologien verändern mit hoher Geschwindigkeit Branchen und Unternehmen: Das Mobile Internet, die Automatisierung der Wissensarbeit, das Internet der Dinge, Cloud Technologien, Advanced Robotics, 3D-Druck, Block Chain usw. Jede dieser Technologien hat das Potential, Branchen von Grund auf zu verändern, und stellt Unternehmen vor die große Herausforderung, Schritt zu halten. Nun stellen Sie sich aber vor, dass diese Technologien branchenübergreifend mehr und mehr kombiniert werden. Früher hatte es gereicht, die eigene Branche 
zu kennen, die Entwicklungen darin zu beobachten, den Wettbewerb im Auge zu behalten und die Technologien zu beherrschen. Das ändert sich. Der Fortschritt ist exponentiell, digital und kombinatorisch. Das ist zwar notwendig, aber nicht hinreichend. Brynjolfsson und McAfee (2014, S. 101) beschreiben das in ihrem Buch „The Second Machine Age“ so: „Ist Innovation tatsächlich ein Rekombinationsphänomen, zeichnet sich ein Problem ab: Wenn die Zahl der Bausteine explodiert, besteht die größte Schwierigkeit darin, zu erkennen, welche Kombinationen daraus jeweils von Wert sind“. Das erfordert Umdenken und neue Methoden. Öffnung, Geschwindigkeit und Innovation werden zur Überlebensfrage.

Funding Open access funding provided by University of Innsbruck and Medical University of Innsbruck.

Open Access Dieser Artikel wird unter der Creative Commons Namensnennung 4.0 International Lizenz (http://creativecommons.org/licenses/by/4.0/deed.de) veröffentlicht, welche die Nutzung, Vervielfältigung, Bearbeitung, Verbreitung und Wiedergabe in jeglichem Medium und Format erlaubt, sofern Sie den/die ursprünglichen Autor(en) und die Quelle ordnungsgemäß nennen, einen Link zur Creative Commons Lizenz beifügen und angeben, ob Änderungen vorgenommen wurden.

\section{Literatur}

BCG (2015). Industry 4.0. The future of producitivity and growth in manufacutring industries

Brynjolfsson, E., \& McAfee, A. (2014). The second machine age: work, progress, and prosperity in a time of brilliant technologies. New York: WW Norton.

Bughin, J., Hazan, E., Labaye, E., Manyika, J., Dahlström, P., Ramaswamy, S., \& Cochin de Billy, C. (2016). Digital Europe: Realizing the continent's potential. New York: McKinsey Global Institute.

Buvat, J., \& Subrahmanyam, K. V.J. (2016). Going up @ Schindlers. Paris: Capgemini Digital Masters series.

Drucker, P. (1963). Managing for Business Effectiveness. Harvard Business Review, 3/1963, 53-60.

Ericsson (2015). Ericsson Mobility Report: 70 percent of world's population using smartphones by 2020. https://www.ericsson.com/news/1925907. Zugegriffen: 20. Jan. 2019.

Evans, P., \& Forth, P. (2015). Navigating a world of digital disruption. IEEE Engineering Management Review, 43(3), 89-97.

Fleisch, E., Weinberger, M., \& Wortmann, F. (2014). Geschäftsmodelle im Internet der Dinge. HMD Praxis der Wirtschaftsinformatik, 51(6), 812-826.

Gassmann, O., Frankenberger, K., \& Csik, M. (2013). Geschäftsmodelle entwickeln: 55 innovative Konzepte mit dem St. Galler Business Model Navigator. München: Hanser.

Keese, C. (2014). Silicon Valley: Was aus dem mächtigsten Tal der Welt auf uns zukommt. München: Albrecht Knaus.

Manyika, J. (2015). The internet of things: mapping the value beyond the hype. New York: McKinsey Global Institute.

Matzler, K., Bailom, F., \& von den Eichen Anschober, F. S. M. (2016). Digital Disruption. Wie Sie Ihr Unternehmen auf das digitale Zeitalter vorbereiten. München: Vahlen.

Meck, G., \& Weiguny, B. (2015). Disruption, Baby, Disruption. Frankfurter Allgemeine Zeitung, 27. Dez. 2015.

Rühl, G. (2016). Wir wollen die Mentalität übertragen. Handelsblatt, 4. Apr. 2016. 


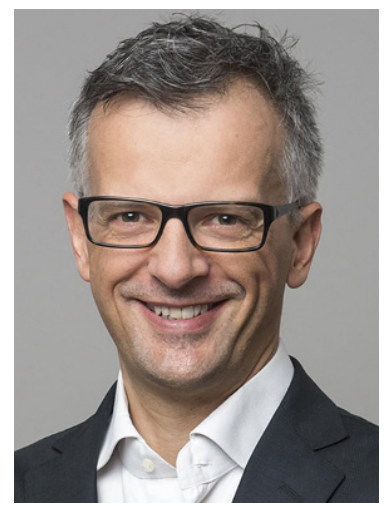

Kurt Matzler Professor für strategisches Management an der Universität Innsbruck, wissenschaftlicher Leiter des Executive MBA am MCI in Innsbruck und Gesellschafter vom Beratungsunternehmen IMP. Er ist Autor von mehr als 300 wissenschaftlichen Artikeln und mehreren Büchern, darunter Ko-Autor der deutschen Ausgabe des Innovator's Dilemma und des Buch „Digital Disruption“. Google Scholar listet ihn unter die Top 20 Strategieprofessoren Europas und unter die Top 50 der Welt.

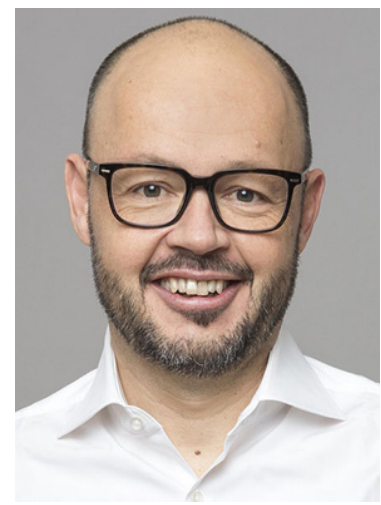

Dr. Franz Bailom Gründer und CEO von in-manas: Intelligent Management Solutions. Mit einem Expertenteam aus unterschiedlichen Disziplinen wurde bei in-manas in den letzten drei Jahren der erste digitale Management-Assistent entwickelt. Unternehmen sollen dadurch in der Strategie- und Innovationsarbeit schneller, agiler und effektiver werden. Der digitale Management-Assistent kommt bereits bei ausgewählten Unternehmen zum Einsatz. 